(C2009 IEEE. Personal use of this material is permitted. However, permission to reprint/republish this material for advertising or promotional purposes or for creating new collective works for resale or redistribution to servers or lists, or to reuse any copyrighted component of this work in other works must be obtained from the IEEE. 


\title{
Asymptotic quotient observers for 2-D Fornasini Marchesini models
}

\author{
Lorenzo Ntogramatzidis and Michael Cantoni
}

\begin{abstract}
The concepts of conditioned-invariant, detectability and input-containing subspaces are developed within the context of observer design for 2-D FornasiniMarchesini models in a general form. Specifically, a link is establised between these subspaces and the existence of so-called quotient observers, which estimate the local state modulo a conditioned invariant subspace. We also consider the synthesis of observers that are asymptotic in the sense that the estimation error (modulo a conditioned invariant subspace) tends to zero away from the boundary values.
\end{abstract}

\section{INTRODUCTION}

Conditioned invariant subspaces for 1-D systems were introduced by Basile and Marro in [1] as the dual of controlled invariant subspaces. The role of such subspaces in relation to the problem of estimation in the presence of unknown input signals was investigated by the same authors in [2]. An alternative definition of conditioned invariance was proposed by Willems in terms of the existence of certain observers [25]; also see the recent textbooks [3, Chapter 4] and [24, Chapter 5].

The purpose of this paper is to first extend the definition of conditioned invariance and input-containing subspaces given for 1-D systems in [1], to Fornasini-Marchesini models [7], [10] in the general form

$$
\begin{aligned}
x_{i+1, j+1}= & A_{0} x_{i, j}+A_{1} x_{i+1, j}+A_{2} x_{i, j+1} \\
& +B_{0} u_{i, j}+B_{1} u_{i+1, j}+B_{2} u_{i, j+1}, \\
y_{i, j}= & C x_{i, j}+D u_{i, j},
\end{aligned}
$$

of Kurek [18]. Our approach to defining conditioned invariant subspaces is similar to that of Willems in that we ultimately seek an observer of the form ${ }^{1}$

$$
\begin{aligned}
\omega_{i+1, j+1}= & K_{0} \omega_{i, j}+K_{1} \omega_{i+1, j}+K_{2} \omega_{i, j+1} \\
& +L_{0} y_{i, j}+L_{1} y_{i+1, j}+L_{2} y_{i, j+1},
\end{aligned}
$$

so that the estimation error $e_{i, j} \triangleq Q x_{i, j}-\omega_{i, j}$, for some full row-rank matrix $Q,{ }^{2}$ asymptotically approaches zero away from standard boundary conditions. To this end, we develop notions of conditioned-invariant, detectability and

\footnotetext{
This work was supported in part by the Australian Research Council (Discovery Grans DP0664789 and DP0986577).

L. Ntogramatzidis is with the Department of Mathematics and Statistics, Curtin University of Technology, Perth WA 6845, Australia 1.ntogramatzidis@curtin.edu.au

Michael Cantoni is with the Department of Electrical and Electronic Engineering, The University of Melbourne, Parkville, VIC 3010, Australia.cantoni@unimelb.edu.au

${ }^{1}$ This is different to the less general form considered in [22].

${ }^{2}$ That is, the error modulo the null space of $Q$.
}

input-containing subspaces, which turn out to be related to the existence of matrices $\Lambda_{i}$ and $\Gamma_{i}$ for which

$$
\begin{aligned}
& Q\left[\begin{array}{lll}
A_{0} & A_{1} & A_{2}
\end{array}\right]-\left[\begin{array}{lll}
\Lambda_{0} & \Lambda_{1} & \Lambda_{2}
\end{array}\right] \operatorname{diag}(C, C, C) \\
& =\left[\begin{array}{lll}
\Gamma_{0} & \Gamma_{1} & \Gamma_{2}
\end{array}\right] \operatorname{diag}(Q, Q, Q) .
\end{aligned}
$$

In this way, when there are no inputs (i.e. $u_{i, j}=0$ ), with $L_{i}=\Lambda_{i}$ and $K_{i}=\Gamma_{i}$ the estimation error satisfies

$$
\begin{aligned}
e_{i+1, j+1}= & \left(Q\left[\begin{array}{lll}
A_{0} & A_{1} & A_{2}
\end{array}\right]\right. \\
& \left.-\left[\begin{array}{lll}
L_{0} & L_{1} & L_{2}
\end{array}\right] \operatorname{diag}(C, C, C)\right)\left[\begin{array}{c}
x_{i, j} \\
x_{i+1, j} \\
x_{i, j+1}
\end{array}\right] \\
& -\left[\begin{array}{lll}
K_{0} & K_{1} & K_{2}
\end{array}\right]\left[\begin{array}{c}
\omega_{i, j} \\
\omega_{i+1, j} \\
\omega_{i, j+1}
\end{array}\right] \\
= & \Gamma_{0} e_{i, j}+\Gamma_{1} e_{i+1, j}+\Gamma_{2} e_{i, j+1} .
\end{aligned}
$$

That is, the dynamics of the estimation error can be expressed as an autonomous FM model in Kurek form. It is interesting to note that, unlike the 1-D case, the required notions of conditioned invariance are not dual of the notion of controlled invariance developed in [6], [21]. This is because the obvious dual of (1) is not in the same form.

Notation. We denote the origin of $\mathbb{R}^{n}$ by $\mathbf{0}_{n}$. The image, kernel, transpose and Moore-Penrose inverse of a matrix $M$ are denoted $\operatorname{im} M, \operatorname{ker} M, M^{\top}$ and $M^{\dagger}$, respectively. The $n \times m$ zero matrix is denoted by $0_{n \times m}$. We define $M_{D} \triangleq$ $\operatorname{diag}(M, M, M)$, and, accordingly, given a subspace $\mathscr{J} \subseteq$ $\mathbb{R}^{n}$, the symbol $\mathscr{J}_{D}$ denotes the subspace $\mathscr{J} \times \mathscr{J} \times \mathscr{J}$ of $\mathbb{R}^{3 n}$, where $\times$ is the Cartesian product. Given the vector $\xi \in \mathbb{R}^{n}$, the symbol $\xi / \mathscr{J}$ denotes the canonical projection of $\xi$ on the quotient space $\mathbb{R}^{n} / \mathscr{J}$. Finally, given a triple of matrices $\left(M_{0}, M_{1}, M_{2}\right)$, we define $M_{H} \triangleq\left[\begin{array}{lll}M_{0} & M_{1} & M_{2}\end{array}\right]$ and $M_{V} \triangleq\left[\begin{array}{lll}M_{0}^{\top} & M_{1}^{\top} & M_{2}^{\top}\end{array}\right]^{\top}$.

\section{INVARIANT SUBSPACES FOR FM MODELS}

We begin by considering the autonomous FM model

$$
x_{i+1, j+1}=A_{0} x_{i, j}+A_{1} x_{i+1, j}+A_{2} x_{i, j+1} .
$$

As boundary conditions for (3) we use $x_{i, j}=b_{i, j} \in \mathbb{R}^{n}$ for all $(i, j) \in \mathfrak{B}$ and some constants $b_{i, j} \in \mathbb{R}^{n}$, where $\mathfrak{B} \triangleq$ $(\{0\} \times \mathbb{N}) \cup(\mathbb{N} \times\{0\}) .^{3}$

A subspace $\mathscr{J}$ of $\mathbb{R}^{n}$ is said to be $\left(A_{0}, A_{1}, A_{2}\right)$-invariant if $\mathscr{J}$ is $A_{i}$-invariant for $i \in\{0,1,2\}$ in the usual 1-D sense; i.e., $A_{i} x \in \mathscr{J}$ for all $x \in \mathscr{J}$ and $i \in\{0,1,2\}$. The following provides geometric and matrix conditions for invariance.

\footnotetext{
${ }^{3}$ Other choices of $\mathfrak{B}$, for which a unique solution of (3) exists, are possible; see [11]. The results in this paper can be adapted to these cases.
} 
Lemma 2.1: The following are equivalent:

1) $\mathscr{J}$ is $\left(A_{0}, A_{1}, A_{2}\right)$-invariant;

2) $A_{H} \mathscr{J}_{D} \subseteq \mathscr{J}$

3) There exist $L_{0}, L_{1}, L_{2} \in \mathbb{R}^{(n-r) \times(n-r)}$ such that $Q A_{i}=$ $L_{i} Q$ for $i \in\{0,1,2\}$, where $Q \in \mathbb{R}^{(n-r) \times n}$ is a full row-rank matrix such that $\operatorname{ker} Q=\mathscr{J}$, i.e., $Q A_{H}=$ $L_{H} Q_{D}$.

Proof: 1) $\Longrightarrow$ 2) For $\xi_{i} \in \mathscr{J}, i \in\{0,1,2\}$, it follows that $A_{i} \xi_{i} \in \mathscr{J}$, and hence, $A_{0} \xi_{0}+A_{1} \xi_{1}+A_{2} \xi_{2}=$ $A_{H}\left[\begin{array}{lll}\xi_{0}^{\top} & \xi_{1}^{\top} & \xi_{2}^{\top}\end{array}\right]^{\top} \in \mathscr{J}$.

2) $\Longrightarrow$ 1) Suppose there exist an $i \in\{0,1,2\}$ and an $\xi \in \mathscr{J}$ such that $A_{i} \xi \notin \mathscr{J}$; i.e., $\mathscr{J}$ is not $\left(A_{0}, A_{1}, A_{2}\right)$ invariant. Then $A_{H}\left[\begin{array}{lll}\xi^{\top} & 0 & 0\end{array}\right]^{\top} \notin \mathscr{J}$, which contradicts 2).

2) $\Leftrightarrow$ 3) Note that 2) is equivalent to $\operatorname{ker} Q_{D} \subseteq \operatorname{ker} Q A_{H}$, by which the result holds, since for any matrices $M \in \mathbb{R}^{p \times m}$ and $N \in \mathbb{R}^{q \times p}$, $\operatorname{ker} M \subseteq \operatorname{ker} N$ if and only if there exist an $L \in \mathbb{R}^{q \times m}$ such that $N=L M$.

The following theorem is the 2-D counterpart of a wellknown result (see [1]) concerning the decomposition of a 1-D system matrix with respect to an invariant subspace.

Theorem 2.1: The following are equivalent:

1) There exists an $r$-dimensional subspace $\mathscr{J} \subseteq \mathbb{R}^{n}$ that is $\left(A_{0}, A_{1}, A_{2}\right)$-invariant;

2) There exists a similarity transformation $S \in \mathbb{R}^{n \times n}$ such that for each $i \in\{0,1,2\}$

$$
\hat{A}_{i} \triangleq S A_{i} S^{-1}=\left[\begin{array}{cc}
\hat{A}_{i}^{11} & \hat{A}_{i}^{12} \\
0_{(n-r) \times r} & \hat{A}_{i}^{22}
\end{array}\right] .
$$

Proof: In view of Lemma 2.1, the proof follows that of Theorem 2.1 in [21], via a similarity transformation $T$ such that $T^{-1}=S$, where $S$ is any non-singular matrix for which $Q S^{-1}=\left[{ }_{(n-r) \times r} I_{(n-r)}\right]$, where $Q$ is a full row-rank matrix such that $\operatorname{ker} Q=\mathscr{V}$. In particular, with respect to the corresponding basis, the identities $Q A_{i}=L_{i} A_{i}$ in 3) of Lemma 2.1 can be expressed as

$$
\left[\begin{array}{ll}
0_{(n-r) \times r} & I_{(n-r)}
\end{array}\right]\left[\begin{array}{cc}
\hat{A}_{i}^{11} & \hat{A}_{i}^{12} \\
0 & \hat{A}_{i}^{22}
\end{array}\right]=L_{i}\left[\begin{array}{ll}
0_{(n-r) \times r} & I_{(n-r)}
\end{array}\right],
$$

by which $L_{i}=\hat{A}_{i}^{22}$.

\section{A. Invariant Subspaces and Local-State Trajectories}

Lemma 2.2: Consider an $\left(A_{0}, A_{1}, A_{2}\right)$-invariant subspace $\mathscr{J}$. A boundary condition $x_{i, j}=b_{i, j} \in \mathscr{J}$, for $(i, j) \in \mathfrak{B}$, gives rise to $x_{i, j} \in \mathscr{J}$ for all $i, j \geq 0$.

Proof: In the set of coordinates corresponding to the similarity transformation $\mathrm{S}$ in Theorem 2.1, it follows that

$$
\begin{aligned}
{\left[\begin{array}{c}
x_{i+1, j+1}^{\prime} \\
x_{i+1, j+1}^{\prime \prime}
\end{array}\right]=} & {\left[\begin{array}{cc}
\hat{A}_{0}^{11} & \hat{A}_{0}^{12} \\
0 & \hat{A}_{0}^{22}
\end{array}\right]\left[\begin{array}{c}
x_{i, j}^{\prime} \\
x_{i, j}^{\prime \prime}
\end{array}\right]+\left[\begin{array}{cc}
\hat{A}_{1}^{11} & \hat{A}_{1}^{12} \\
0 & \hat{A}_{1}^{22}
\end{array}\right]\left[\begin{array}{c}
x_{i+1, j}^{\prime} \\
x_{i+1, j}^{\prime \prime}
\end{array}\right] } \\
& +\left[\begin{array}{cc}
\hat{A}_{2}^{11} & \hat{A}_{2}^{12} \\
0 & \hat{A}_{2}^{22}
\end{array}\right]\left[\begin{array}{c}
x_{i, j+1}^{\prime} \\
x_{i, j+1}^{\prime \prime}
\end{array}\right] .
\end{aligned}
$$

Note that any boundary condition $x_{i, j}=b_{i, j} \in \mathscr{J}$ is such that $x_{i, j}^{\prime \prime}=0$ for $(i, j) \in \mathfrak{B}$. Moreover, by (5), $x_{i, j}^{\prime \prime}=0$ for all $i, j \geq 0$. Hence, $x_{i, j} \in \mathscr{J}$ for all $i, j \geq 0$.
In the basis corresponding to $S$ in the proof of Lemma 2.2, whereby $\left[\begin{array}{c}x_{i, j}^{\prime} \\ x_{i, j}^{\prime \prime}\end{array}\right]=S x_{i, j}$, the component $x_{i, j}^{\prime}$ is the projection of the local state $x_{i, j}$ onto the invariant subspace $\mathscr{J}$, while $x_{i, j}^{\prime \prime}$ is the canonical projection on to the quotient space $\mathbb{R}^{n} / \mathscr{J}$.

\section{B. Internal and External Stability of Invariant Subspaces}

A necessary and sufficient condition for asymptotic stability of (3) - often said asymptotic stability of the triple $\left(A_{0}, A_{1}, A_{3}\right)-$ is that $\forall\left(z_{1}, z_{2}\right) \in \mathfrak{P}$

$$
\operatorname{det}\left(I_{n}-A_{0} z_{1} z_{2}-A_{1} z_{2}-A_{2} z_{1}\right) \neq 0,
$$

where $\mathfrak{P} \triangleq\left\{\left(\zeta_{1}, \zeta_{2}\right) \in \mathbb{C} \times \mathbb{C}|| \zeta_{1} \mid<1\right.$ and $\left.\left|\zeta_{2}\right|<1\right\}$; this is equivalent to $x_{i, j} \rightarrow 0$ as $i+j \rightarrow \infty$. Various, more computationally tractable, sufficient stability conditions have been proposed over the last two decades, in terms of Lyapunov equations and/or spectral radius conditions of certain matrices, see e.g. [14], [5]. In the very recent literature, new necessary and sufficient criteria have appeared for asymptotic stability in terms of conditions that can be checked in finite terms, see [27], [9]. For the sake of argument and clarity, however, the following simple sufficient condition for asymptotic stability, expressed in terms of an linear matrix inequality (LMI), will be used herein:

Lemma 2.3: ([14]) The triple $\left(A_{0}, A_{1}, A_{2}\right)$ is asymptotically stable if three symmetric positive definite matrices $P_{0}, P_{1}$ and $P_{2}$ exist such that:

$$
\operatorname{diag}\left(P_{0}, P_{1}, P_{2}\right)-A_{H}^{\top}\left(P_{0}+P_{1}+P_{2}\right) A_{H}>0 .
$$

We now show that stability of (3) can be "split" into two parts with respect to an invariant subspace $\mathscr{J} \subseteq \mathbb{R}^{n \times n}$. Expressing (3) in the set of coordinates corresponding to the similarity transformation $S$ in Theorem 2.1,

$$
\begin{aligned}
\operatorname{det}\left(I_{n}-\right. & \left.\hat{A}_{0} z_{1} z_{2}-\hat{A}_{1} z_{2}-\hat{A}_{2} z_{1}\right) \\
= & \operatorname{det}\left(I-\hat{A}_{0}^{11} z_{1} z_{2}-\hat{A}_{1}^{11} z_{2}-\hat{A}_{2}^{11} z_{1}\right) \\
& \cdot \operatorname{det}\left(I-\hat{A}_{0}^{22} z_{1} z_{2}-\hat{A}_{1}^{22} z_{2}-\hat{A}_{2}^{22} z_{1}\right) .
\end{aligned}
$$

It follows that (3) is asymptotically stable if and only if $\left(\hat{A}_{0}^{11}, \hat{A}_{1}^{11}, \hat{A}_{2}^{11}\right)$ and $\left(\hat{A}_{0}^{22}, \hat{A}_{1}^{22}, \hat{A}_{2}^{22}\right)$ are asymptotically stable.

Definition 2.1: The $\left(A_{0}, A_{1}, A_{2}\right)$-invariant subspace $\mathscr{J}$ is

- internally stable if the corresponding triple $\left(\hat{A}_{0}^{11}, \hat{A}_{1}^{11}, \hat{A}_{2}^{11}\right)$ is asymptotically stable.

- externally stable if the corresponding triple $\left(\hat{A}_{0}^{22}, \hat{A}_{1}^{22}, \hat{A}_{2}^{22}\right)$ is asymptotically stable.

Hence, (3) is asymptotically stable if and only if any invariant subspace is both internally and externally stable.

Corollary 2.1: Given a subspace $\mathscr{J}$ of $\mathbb{R}^{n}$, let $Q \in$ $\mathbb{R}^{(n-r) \times n}$ be a full row-rank matrix such that $\operatorname{ker} Q=$ $\mathscr{J}$. Then $\mathscr{J}$ is an externally stable $\left(A_{0}, A_{1}, A_{2}\right)$-invariant subspace if and only if an asymptotically stable triple $\left(L_{0}, L_{1}, L_{2}\right)$ exists such that $Q A_{i}=L_{i} Q$ for all $i \in\{0,1,2\}$. 
Proof: See last part of the proof of Theorem 2.1, whereby $\hat{A}_{i}^{22}=L_{i}$.

\section{CONDITIONED INVARIANT SUbSPACES}

Now we focus on the definition of conditioned invariant subspaces for (1). Such subspaces are shown to be related to the existence of a so-called quotient observer as discussed in the introduction.

Definition 3.1: The subspace $\mathscr{S} \subseteq \mathbb{R}^{n}$ is conditioned invariant for (1) if $A_{H}\left(\mathscr{S}_{D} \cap \operatorname{ker} C_{D}\right) \subseteq \mathscr{S}$.

It is can be seen that the set of conditioned invariant subspaces is closed under subspace intersection. Its smallest element is $\mathbf{0}_{n}$, its largest element is $\mathbb{R}^{n}$.

Lemma 3.1: Let $\mathscr{S}$ be an $s$-dimensional subspace of $\mathbb{R}^{n}$, and let $Q \in \mathbb{R}^{(n-s) \times n}$ be such that ker $Q=\mathscr{S}$ with $Q$ of full row-rank. The following statements are equivalent:

1) the subspace $\mathscr{S}$ is conditioned invariant for (1);

2) there exist matrices $\Gamma=\left[\Gamma_{0} \Gamma_{1} \Gamma_{2}\right] \in \mathbb{R}^{(n-s) \times 3(n-s)}$ and $\Lambda=\left[\begin{array}{lll}\Lambda_{0} & \Lambda_{1} & \Lambda_{2}\end{array}\right] \in \mathbb{R}^{(n-s) \times 3 p}$ such that

$$
Q A_{H}=\Gamma Q_{D}+\Lambda C_{D}
$$

3) there exist a matrix $G=\left[G_{0} G_{1} G_{2}\right] \in \mathbb{R}^{n \times 3 p}$ such that

$$
\left(A_{H}+G C_{D}\right) \mathscr{S}_{D} \subseteq \mathscr{S} \text {. }
$$

Proof: 1) $\Longrightarrow$ 2). Since $\mathscr{S}$ is such that $A_{H}\left(\mathscr{S}_{D} \cap\right.$ $\left.\operatorname{ker} C_{D}\right) \subseteq \mathscr{S}$, it follows that $\operatorname{ker}\left[\begin{array}{l}Q_{D} \\ C_{D}\end{array}\right] \subset \operatorname{ker} Q A_{H}$ and as such, there exist $\Gamma \in \mathbb{R}^{(n-s) \times 3(n-s)}$ and $\Lambda \in \mathbb{R}^{(n-s) \times 3 p}$ such that $Q A_{H}=\Gamma Q_{D}+\Lambda C_{D}$; see Proof of Lemma 2.1. 2) $\Longrightarrow$ 3). Equation (9) follows from (8) with any $G$ such that $\Lambda=-Q G$. 3) $\Longrightarrow$ 1). This follows by defintion.

Property 3) in Lemma 3.1 means that $\mathscr{S}$ is conditioned invariant for (1) if and only if there exists an outputinjection matrix $G=\left[G_{0} G_{1} G_{2}\right] \in \mathbb{R}^{n \times 3 p}$ such that $\mathscr{S}$ is a $\left(A_{0}+G_{0} C, A_{1}+G_{1} C, A_{2}+G_{2} C\right)$-invariant subspace. Let $\Gamma$ and $\Lambda$ be such that (8) holds, which can be written as

$$
Q A_{H}=\left[\begin{array}{ll}
\Gamma & \Lambda
\end{array}\right]\left[\begin{array}{l}
Q_{D} \\
C_{D}
\end{array}\right],
$$

a linear equation which can be solve for $\Gamma$ and $\Lambda$. Given a conditioned invariant subspace, the solutions of (10) are given by

$$
\left[\begin{array}{ll}
\Gamma & \Lambda
\end{array}\right]=Q A_{H}\left[\begin{array}{l}
Q_{D} \\
C_{D}
\end{array}\right]^{\dagger}+K H,
$$

where the rows of $H$ span the null-space of $\left[\begin{array}{ll}Q_{D}^{\top} & C_{D}^{\top}\end{array}\right]$ and $K$ is an arbitrary matrix of suitable size. When $\left[\begin{array}{l}Q_{D} \\ C_{D}\end{array}\right]$ is full-rank, matrix $K$ has zero rows; i.e., the only solution of (10) is $[\Gamma \Lambda]=Q A_{H}\left[\begin{array}{l}Q_{D} \\ C_{D}\end{array}\right]^{\dagger}$. By (9), $\tilde{\Gamma}$ exists such that

$$
Q\left(A_{H}+G C_{D}\right)=\tilde{\Gamma} Q_{D} .
$$

We now investigate the relation between the pairs $(\Gamma, \Lambda)$ and $(G, \tilde{\Gamma})$ satisfying (10) and (12), respectively. First, notice that Given a pair $(G, \tilde{\Gamma})$ such that (12) holds, then (10) is satisfied with $\Gamma=\tilde{\Gamma}$ and $\Lambda=-Q G$. Conversely, given a pair of matrices $(\Gamma, \Lambda)$ such that (10) holds, then (12) is satisfied with $\tilde{\Gamma}=\Gamma$ and with any $G$ such that $\Lambda=-Q G$. As such, no generality is lost by assuming $\tilde{\Gamma}=\Gamma$, and by representing the set of all friends of the conditioned invariant subspace $\mathscr{S}$ as the set of matrices $G \in \mathbb{R}^{n \times 3 p}$ satisfying $\Lambda=-Q G$, where $\Lambda \in \mathbb{R}^{(n-s) \times 3 p}$ is any matrix for which another matrix $\Gamma \in \mathbb{R}^{(n-s) \times 3(n-s)}$ exists so that (10) holds. For any pair $(\Gamma, \Lambda)$ such that (10) holds, the solutions of the linear equation $\Lambda=-Q G$ are parameterised as

$$
G=G_{\Lambda}+\tilde{G}
$$

where $G_{\Lambda} \triangleq-Q^{\top}\left(Q Q^{\top}\right)^{-1} \Lambda$ and $\tilde{G}$ is any $n \times 3 p$ matrix such that $Q \tilde{G}=0$, or, equivalently, such that $\operatorname{im} \tilde{G} \subseteq \operatorname{ker} Q$. The choice of $\tilde{G}$ affects the external stability of $\mathscr{S}$, but not the internal stability of $\mathscr{S}$. Similarly, $G_{\Lambda}$ can affect the internal but not the external stability of $\mathscr{S}$. With reference to the proof of Corollary 2.1, note that with $S \triangleq\left[\begin{array}{c}S_{c} \\ Q\end{array}\right]$, where the rows of $S_{c}$ are linearly independent from those of $Q$, so that $Q S^{-1}=[0 I]$, we have that for all $i \in\{0,1,2\}$

$$
S\left(A_{i}+G_{i} C\right) S^{-1}=\left[\begin{array}{cc}
\Delta_{i}^{11}(\Lambda, \tilde{G}) & \Delta_{i}^{12}(\Lambda, \tilde{G}) \\
0 & \Delta_{i}^{22}(\Lambda, \tilde{G})
\end{array}\right]
$$

Lemma 3.2: For all $i \in\{0,1,2\}$, the matrix $\Delta_{i}^{22}(\Lambda, \tilde{G})$ does not depend on $\tilde{G}$, and the matrix $\Delta_{i}^{11}(\Lambda, \tilde{G})$ does not depend on the particular $\Lambda$ which satisfies (10) for some $\Gamma$.

Proof: First, let $\tilde{G}_{1}, \tilde{G}_{2}$ be such that $Q \tilde{G}_{1}=0$ and $Q \tilde{G}_{2}=$ 0 . From (14) we find that

$$
\begin{aligned}
& \quad\left[\begin{array}{cc}
\Delta_{i}^{11}\left(\Lambda, \tilde{G}_{1}\right)-\Delta_{i}^{11}\left(\Lambda, \tilde{G}_{2}\right) & \Delta_{i}^{12}\left(\Lambda, \tilde{G}_{1}\right)-\Delta_{i}^{12}\left(\Lambda, \tilde{G}_{2}\right) \\
0 & \Delta_{i}^{22}\left(\Lambda, \tilde{G}_{1}\right)-\Delta_{i}^{22}\left(\Lambda, \tilde{G}_{2}\right)
\end{array}\right] \\
& =S\left(A_{i}+G_{\Lambda, i} C+\tilde{G}_{1}\right) S^{-1}-S\left(A_{i}+G_{\Lambda, i} C+\tilde{G}_{2}\right) S^{-1} \\
& =\left[\begin{array}{c}
S_{c} \\
Q
\end{array}\right]\left(\tilde{G}_{1}-\tilde{G}_{2}\right) C S^{-1},
\end{aligned}
$$

so that

$$
\begin{aligned}
& Q\left(\tilde{G}_{1}-\tilde{G}_{2}\right) C_{i} T^{-1} \\
= & {\left[\begin{array}{ll}
0 & \Delta_{i}^{22}\left(\Lambda, \tilde{G}_{1}\right)-\Delta_{i}^{22}\left(\Lambda, \tilde{G}_{2}\right)
\end{array}\right]=0, }
\end{aligned}
$$

since $Q \tilde{G}_{1}=0$ and $Q \tilde{G}_{2}=0$.

Now, from (14), it is follows that

$$
\begin{array}{r}
S_{c}\left(A_{i}-Q^{\top}\left(Q Q^{\top}\right)^{-1} \Lambda C+\tilde{G} C\right) \\
\quad=\Delta_{i}^{11}(\Lambda, \tilde{G}) S_{c}+\Delta_{i}^{12}(\Lambda, \tilde{G}) Q .
\end{array}
$$

Let $\Lambda_{a}$ and $\Lambda_{b}$ such that $Q A_{H}=\Gamma_{\star} Q_{D}+\Lambda_{\star} \hat{C}$, for $\star \epsilon$ $\{a, b\}$. The difference of these equations leads to $\left(\Lambda_{a}-\right.$ $\left.\Lambda_{b}\right) C_{D}=-\left(\Gamma_{a}-\Gamma_{b}\right) Q_{D}$. By partitioning $\left(\Gamma_{a}-\Gamma_{b}\right)$ as $\left[\begin{array}{lll}\Xi_{0} & \Xi_{1} & \Xi_{2}\end{array}\right]$, we get $\left(\Lambda_{a}-\Lambda_{b}\right) C_{D}=-\Xi_{i} Q$. Writing (15) with respect to $\Lambda_{a}$ and $\Lambda_{b}$ and by computing the difference 
yields

$$
\begin{aligned}
& S_{c}\left(-Q^{\top}\left(Q Q^{\top}\right)^{-1}\left(\Lambda_{a}-\Lambda_{b}\right) C_{D}\right) \\
= & \left(\Delta_{i}^{11}\left(\Lambda_{a}, \tilde{G}\right)-\Delta_{i}^{11}\left(\Lambda_{b}, \tilde{G}\right)\right) S_{c} \\
& +\left(\Delta_{i}^{12}\left(\Lambda_{a}, \tilde{G}\right)-\Delta_{i}^{12}\left(\Lambda_{b}, \tilde{G}\right)\right) Q,
\end{aligned}
$$

so that

$$
\begin{aligned}
& S_{c} Q^{\top}\left(Q Q^{\top}\right)^{-1} \Delta_{i} Q \\
= & \left(\Delta_{i}^{11}\left(\Lambda_{a}, \tilde{G}\right)-\Delta_{i}^{11}\left(\Lambda_{b}, \tilde{G}\right)\right) S_{c} \\
& +\left(\Delta_{i}^{12}\left(\Lambda_{a}, \tilde{G}\right)-\Delta_{i}^{12}\left(\Lambda_{b}, \tilde{G}\right)\right) Q .
\end{aligned}
$$

Since $Q$ and $S_{c}$ have linearly independent rows, we find

$$
S_{c} Q^{\top}\left(Q Q^{\top}\right)^{-1} \Xi_{i} Q=\left(\Delta_{i}^{12}\left(\Lambda_{a}, \tilde{G}\right)-\Delta_{i}^{12}\left(\Lambda_{b}, \tilde{G}\right)\right) Q
$$

and

$$
\left(\Delta_{i}^{11}\left(\Lambda_{a}, \tilde{G}\right)-\Delta_{i}^{11}\left(\Lambda_{b}, \tilde{G}\right)\right) S_{c}=0,
$$

the second yielding $\Delta_{i}^{11}\left(\Lambda_{a}, \tilde{G}\right)=\Delta_{i}^{11}\left(\Lambda_{b}, \tilde{G}\right)$ since $S_{c}$ has linearly independent rows.

Conditioned invariance is linked to the exisitence of 2-D quotient observers [22]. For an observer of the form (2) for (1) with $u_{i, j}=0,{ }^{4}$ it follows that with $e_{i, j}:=Q x_{i, j}-\omega_{i, j}$,

$$
e_{i+1, j+1}=\left(Q A_{H}-L_{H} C_{D}\right)\left[\begin{array}{c}
x_{i, j} \\
x_{i+1, j} \\
x_{i, j+1}
\end{array}\right]+K_{H}\left[\begin{array}{c}
\omega_{i, j} \\
\omega_{i+1, j} \\
\omega_{i, j+1}
\end{array}\right] .
$$

For $K_{H}=\Gamma$ and $L_{H}=\Lambda$, where $(\Lambda, \Gamma)$ satisfy (10), this becomes

$$
\begin{aligned}
e_{i+1, j+1} & =\Gamma Q_{D}\left[\begin{array}{c}
x_{i, j} \\
x_{i+1, j} \\
x_{i, j+1}
\end{array}\right]+\Gamma\left[\begin{array}{c}
\omega_{i, j} \\
\omega_{i+1, j} \\
\omega_{i, j+1}
\end{array}\right] \\
& =\Gamma_{0} e_{i, j}+\Gamma_{1} e_{i+1, j}+\Gamma_{2} e_{i, j+1},
\end{aligned}
$$

so that with observer boundary conditions $\omega_{i, j}=x_{i, j} / \mathscr{S}$, for $(i, j) \in \mathfrak{B}$, it follows that $e_{i, j}=0$ for $(i, j) \in \mathfrak{B}$, and hence, all $(i, j) \in \mathbb{N} \times \mathbb{N}$ by (17). If $\left(\Gamma_{0}, \Gamma_{1}, \Gamma_{2}\right)$ is asymptotically stable, then the observer is said to be asymptotic in the sense that $e_{i, j} \rightarrow 0$ as $i+j \rightarrow \infty$ for any boundary conditions. In view of Corollary 2.1, part 2), we are therefore interested in finding $G=\left[\begin{array}{lll}G_{0} & G_{1} G_{2}\end{array}\right]$ such that $\mathscr{S}$ is an externally stable $\left(A_{0}+G_{0} C, A_{1}+\right.$ $G_{1} C, A_{2}+G_{2} C$ )-invariant subspace; i.e., such that there exists an asymptotically stable triple $\left(\Gamma_{0}, \Gamma_{1}, \Gamma_{2}\right)$ for which $Q\left(A_{H}+G C_{D}\right)=\Gamma Q_{D}$. When such a $G$ exists, $\mathscr{S}$ is called a detectability subspace.

For a given conditioned invariant $\mathscr{S}$, write (11) as

$\left[\begin{array}{llll}\Gamma_{0} & \Gamma_{1} & \Gamma_{2} & \Lambda\end{array}\right]=\left[\begin{array}{llll}V_{0} & V_{1} & V_{2} & V_{3}\end{array}\right]+K\left[\begin{array}{llll}H_{0} & H_{1} & H_{2} & H_{3}\end{array}\right]$, where $\left[\begin{array}{llll}V_{0} & V_{1} & V_{2} & V_{3}\end{array}\right]=Q A_{H}\left[\begin{array}{l}Q_{D} \\ C_{D}\end{array}\right]^{\dagger}$ and the rows of $\left[\begin{array}{llll}H_{0} & H_{1} & H_{2} & H_{3}\end{array}\right]$, partitioned comformably with $\left[\begin{array}{llll}\Gamma_{0} & \Gamma_{1} & \Gamma_{2} & \Lambda\end{array}\right]$, span the kernel of $\left[\begin{array}{ll}Q_{D}^{\top} & C_{D}^{\top}\end{array}\right]$. If this null space is zero, i.e., if $\mathscr{S}_{D}+\operatorname{ker} C_{D}=\mathbb{R}^{3 n}$, there is

\footnotetext{
${ }^{4}$ Recall that this is more general than the form considered in [22].
}

only one solution to (10), so that there are no degrees of freedom in the choice of the triple $\left(\Gamma_{0}, \Gamma_{1}, \Gamma_{2}\right)$. In this case, if $\left(\Gamma_{0}, \Gamma_{1}, \Gamma_{2}\right)=\left(V_{0}, V_{1}, V_{2}\right)$ is stable, then with the corresponding $\Lambda=\left[\begin{array}{lll}\Lambda_{0} & \Lambda_{1} & \Lambda_{2}\end{array}\right]=V_{3}$, the matrix $G_{\Lambda} \triangleq$ $-Q^{\top}\left(Q Q^{\top}\right)^{-1} \Lambda=\left[G_{\Lambda, 0} G_{\Lambda, 1} G_{\Lambda, 2}\right]$ is such that $\mathscr{S}$ is an externally stable $\left(A_{0}+G_{\Lambda, 0} C, A_{1}+G_{\Lambda, 1} C, A_{2}+G_{\Lambda, 2} C\right)$ invariant subspace. On the other hand, if the triple $\left(\Gamma_{0}, \Gamma_{1}, \Gamma_{2}\right)=\left(V_{0}, V_{1}, V_{2}\right)$ is not asymptotically stable, the subspace $\mathscr{S}$ is not a detectability subspace.

Now, when $\mathscr{S}_{D}+\operatorname{ker} C_{D} \subset \mathbb{R}^{3 n}$, the problem we need to solve is to find a matrix $K$ such that the resulting triple $\left(\Gamma_{0}, \Gamma_{1}, \Gamma_{2}\right)=\left(V_{0}+K H_{0}, V_{1}+K H_{1}, V_{2}+K H_{2}\right)$ is asymptotically stable; the corresponding $\Lambda=\left[\Lambda_{0} \Lambda_{1} \Lambda_{2}\right]=$ $V_{3}+K H_{3}$, for which $(\Gamma, \Lambda)$ is a solution of (8), is such that $G_{\Lambda} \triangleq-Q^{\top}\left(Q Q^{\top}\right)^{-1} \Lambda$, yielding $Q\left(A_{H}+G_{\Lambda} C_{D}\right)=$ $\Gamma Q_{D}$, so that $\mathscr{S}$ is an externally stable $\left(A_{0}+G_{\Lambda, 0} C, A_{1}+\right.$ $\left.G_{\Lambda, 1} C, A_{2}+G_{\Lambda, 2} C\right)$-invariant subspace. Towards characterising a subset of such matrices $K$, we can rewrite the sufficient condition for asymptotic stability in Lemma 2.3 for the triple $\left(\Gamma_{0}, \Gamma_{1}, \Gamma_{2}\right)$ as shown below

$$
\left[\begin{array}{ccc}
\Phi & 0 & 0 \\
0 & \Psi & 0 \\
0 & 0 & \Theta-\Phi-\Psi
\end{array}\right]-\left[\begin{array}{c}
\Gamma_{0}^{\top} \\
\Gamma_{1}^{\top} \\
\Gamma_{2}^{\top}
\end{array}\right] \Theta\left[\begin{array}{ccc}
\Gamma_{0} & \Gamma_{1} & \Gamma_{2}
\end{array}\right]>0,
$$

for some $\Phi \triangleq P_{0}>0, \Psi \triangleq P_{1}>0$ and $\Theta \triangleq P_{0}+P_{1}+P_{2}>0$. Standard manipulation and $\Gamma_{i}=V_{i}+K H_{i}$, for $i=0,1,2$, yield the equivalent condition

$$
\left[\begin{array}{cccc}
\Phi & 0 & 0 & \tilde{V}_{i}^{\top} \\
0 & \Psi & 0 & \tilde{V}_{1}^{\top} \\
0 & 0 & \Theta-\Phi-\Psi & \tilde{V}_{2}^{\top} \\
\tilde{V}_{0} & \tilde{V}_{1} & \tilde{V}_{2} & \Theta
\end{array}\right]>0
$$

for some $\Phi>0, \Psi>0, \Theta>0$ and $\Pi$ of suitable dimensions, where $\tilde{V}_{i} \triangleq \Theta V_{i}+\Pi H_{i}$ and $\Pi=\Theta K$.

Theorem 3.1: Let $\mathscr{S}$ be a conditioned invariant subspace for (1), $\left[\begin{array}{llll}V_{0} & V_{1} & V_{2} & V_{3}\end{array}\right]=Q A_{H}\left[\begin{array}{l}Q_{D} \\ C_{D}\end{array}\right]^{\dagger}$ and $\left[\begin{array}{llll}H_{0} & H_{1} & H_{2} & H_{3}\end{array}\right]$ be such that its rows are a basis for the kernel of $\left[\begin{array}{ll}Q_{D}^{\top} & C_{D}^{\top}\end{array}\right]$. The subspace $\mathscr{S}$ is a detectability subspace if there exist $\Phi=\Phi^{\top}>0, \Psi=\Psi^{\top}>0, \Theta=$ $\Theta^{\top}>0$ and $\Pi$ of suitable dimensions such that (18) holds.

Moreover, given a quadruple $(\Theta, \Phi, \Psi, \Pi)$ in the convex set defined by (18), a matrix $K$ for which the triple $\left(\Gamma_{0}, \Gamma_{1}, \Gamma_{2}\right)$ is asymptotically stable is given by $K=$ $\Theta^{-1} \Pi^{\top}$.

\section{InPUT-CONTAINING SubSPACES}

Now we turn our attention to input-containing subspaces, which are particular types of conditioned invariant subspaces useful in the context of various filtering/estimation problems, like unknown-input observation [22].

Definition 4.1: We define a input-containing subspace $\mathscr{S}$ for (1) as a subspace of $\mathbb{R}^{n}$ such that

$$
\left[\begin{array}{ll}
A_{H} & B_{H}
\end{array}\right]\left(\left(\mathscr{S}_{D} \times \mathbb{R}^{3 m}\right) \cap \operatorname{ker}\left[\begin{array}{ll}
C_{D} & D_{D}
\end{array}\right]\right) \subseteq \mathscr{S} .
$$


The set of input-containing subspaces for (1) is denoted by the symbol $\mathfrak{S}_{0}$. The intersection of two inputcontaining subspaces is input-containing. It follows that the set $\mathfrak{S}_{0}$ is closed under subspace intersection. The same is not true for subspace addition. The intersection of all the input-containing subspaces of $\Sigma$ is the smallest inputcontaining subspace of $\Sigma$, and is usually denoted by $\mathscr{S}^{\star}$.

Lemma 4.1: Given the $s$-dimensional subspace $\mathscr{S}$ of $\mathbb{R}^{n}$, let $Q \in \mathbb{R}^{(n-s) \times n}$ be such that $\operatorname{ker} Q=\mathscr{S}$ with $Q$ of full row-rank. The following statements are equivalent:

1) the subspace $\mathscr{S}$ is input-containing for (1);

2) two matrices $\Gamma \in \mathbb{R}^{(n-s) \times 3(n-s)}$ and $\Lambda \in \mathbb{R}^{(n-s) \times 3 p}$ exist such that

$$
Q\left[\begin{array}{ll}
A_{H} & B_{H}
\end{array}\right]=\Gamma\left[\begin{array}{ll}
Q_{D} & 0
\end{array}\right]+\Lambda\left[\begin{array}{ll}
C_{D} & D_{D}
\end{array}\right] ;
$$

3) a matrix $G \in \mathbb{R}^{n \times 3 p}$ exists such that

$$
\left[\begin{array}{cc}
A_{H}+G C_{D} & B_{H}+G D_{D}
\end{array}\right]\left(\mathscr{S}_{D} \times \mathbb{R}^{3 m}\right) \subseteq \mathscr{S}
$$

Proof: The result follows in the same way as the result in Lemma 3.1.

As before, given an input-containing subspace it is not difficult to see that there exists a quotient observer of the form (2) for (1) in the presence of unkown inputs (possibly non-zero). In particular, it follows that

$$
\begin{gathered}
e_{i+1, j+1}=Q A_{H}\left[\begin{array}{c}
x_{i, j} \\
x_{i+1, j} \\
x_{i, j+1}
\end{array}\right]+Q B_{H}\left[\begin{array}{c}
u_{i, j} \\
u_{i+1, j} \\
u_{i, j+1}
\end{array}\right]-\Gamma\left[\begin{array}{c}
\omega_{i, j} \\
\omega_{i+1, j} \\
\omega_{i, j+1}
\end{array}\right] \\
-Q G C_{D}\left[\begin{array}{c}
x_{i, j} \\
x_{i+1, j} \\
x_{i, j+1}
\end{array}\right]-Q G D_{D}\left[\begin{array}{c}
u_{i, j} \\
u_{i+1, j} \\
u_{i, j+1}
\end{array}\right] \\
\left.=Q\left[A_{H}+G C_{D} \quad B_{H}+G D_{D}\right]\left[\begin{array}{c}
x_{i, j} \\
x_{i, 1, j} \\
u_{i, j} \\
u_{i+1, j} \\
u_{i, j+1}
\end{array}\right]\right]-\Gamma \hat{\omega}(i, j) \\
=\Gamma_{0} e_{i, j}+\Gamma_{1} e_{i+1, j}+\Gamma_{2} e_{i, j+1},
\end{gathered}
$$

where (20) has been used. Moreover if the inputcontaining subspace is a detectability subspace then the observer is asymptotic.

The following is an algorithm for computing the smallest input-containing subspace $\mathscr{S}^{\star}$.

Algorithm 4.1: The sequence of subspaces $\left(\mathscr{S}^{i}\right)_{i \in \mathbb{N}}$ described by the recurrence

$$
\begin{aligned}
\mathscr{S}^{0} & =0_{n} \\
\mathscr{S}^{i} & =\left[\begin{array}{ll}
A_{H} & B_{H}
\end{array}\right]\left(\left(\mathscr{S}_{D}^{i-1} \times \mathbb{R}^{3 m}\right) \cap \operatorname{ker}\left[\begin{array}{ll}
C_{D} & D_{D}
\end{array}\right]\right),
\end{aligned}
$$

for $i>0$, is monotonically non-increasing. An integer $k \leq n-1$ exists such that $\mathscr{S}^{k+1}=\mathscr{S}^{k}$. For such $k$, the identity $\mathscr{S}^{\star}=\mathscr{S}^{k}$ holds.

\section{REFERENCES}

[1] G. Basile and G. Marro. Controlled and conditioned invariant subspaces in linear system theory. Journal of Optimization Theory and Applications, vol. 3, no. 5, pp. 306-315, May 1969.

[2] G. Basile and G. Marro. On the observability of linear, timeinvariant systems with unknown inputs. Journal of Optimization Theory and Applications, vol. 3, no. 6, pp. 410-415, June 1969.
[3] G. Basile and G. Marro. Controlled and Conditioned Invariants in Linear System Theory. Prentice Hall, Englewood Cliffs, New Jersey, 1992.

[4] M. Bisiacco and M.E. Valcher. Unknown input observers for 2D state-space models. International Journal of Control, vol. 77, no. 9, pp. 861-876(16), June 10, 2004.

[5] P.A. Bliman. Lyapunov Equation for the Stability of 2-D Systems. Multidimensional Systems and Signal Processing, vol. 13, pp. 201222, 2002.

[6] G. Conte and A. Perdon. A geometric approach to the theory of 2D systems. IEEE Transactions on Automatic Control, vol. AC-33, no. 10, pp. 946-950, October 1988.

[7] E. Fornasini and G. Marchesini. State-space realization theory of two-dimensional filters. IEEE Transactions on Automatic Control, vol. AC-21, no. 4, pp. 484-492, August 1976.

[8] E. Fornasini and G. Marchesini. Two dimensional filters: New aspects of the realization theory. Proceedings of the 3rd Conference on Pattern Recognition, Coronado, CA, 1976.

[9] Y. Ebihara, and Y. Ito, and T. Hagiwara, Exact Stability Analysis of 2-D Systems Using LMIs. IEEE Transactions on Automatic Control, vol. AC-51, no. 9, pp. 1509-1513, September 2006.

[10] E. Fornasini and G. Marchesini. Doubly-Indexed Dynamical Systems: State-Space Models and Structural Properties. Mathematical System Theory, vol. 12, pp. 59-72, 1978.

[11] E. Fornasini and G. Marchesini, Stability analysis of 2-D systems. IEEE Transactions on Circuits and Systems, CAS-27(12):1210$1217,1980$.

[12] G.D. Hu and M. Liu. Simple Criteria for Stability of TwoDimensional Linear Systems. IEEE Transactions on Signal Processing, vol. 53, no. 12, pp. 4720-4723, December 2005.

[13] T. Kaczorek. The singular general model of 2-D systems and its solution. IEEE Transactions on Automatic Control, vol. AC-33, pp. 1060-1061, 1988.

[14] H. Kar and V. Sigh. Stability of 2-D systems described by the Fornasini-Marchesini first model. IEEE Transactions on Signal Processing, vol. 51, no. 6, pp. 1675-1676, June 2003.

[15] A. Karamanciog̃lu and F.L. Lewis. A geometric approach to 2-D implicit systems. Proceedings of the 29th Conference on Decision and Control, Honolulu, Hawaii, December 1990.

[16] A. Karamanciog̃lu and F.L. Lewis. Geometric theory for the singular Roesser model. IEEE Transactions on Automatic Control, vol. AC-37, no. 6, pp. 801-806, June 1992.

[17] S.-Y. Kung, B.C. Levy, M. Morf, and T. Kailath, New results in 2-D systems theory, Part II: 2-D state-space models - realization and the notions of controllability, observability and minimality, Proceedings of the IEEE, vol. 65, no. 6, pp. 945-961, June 1977.

[18] J. Kurek. The general state-space model for a two-dimensional linear digital system. IEEE Transactions on Automatic Control, vol. AC-30, no. 6, pp. 600-602, June 1985.

[19] F.L. Lewis. A review of 2-D Implicit Systems. Automatica, vol. 28, no. 2, pp. 345-354, 1992.

[20] A.S. Morse. Structure and design of linear model following systems. IEEE Transactions on Automatic Control, vol. AC-18, no. 4, pp. 346-354, August 1973.

[21] L. Ntogramatzidis, M. Cantoni, and R. Yang, A geometric theory for 2-D systems including notions of stabilisability. Multidimensional Systems and Signal Processing, vol.19, pp. 449-475, 2008.

[22] L. Ntogramatzidis and M. Cantoni, Conditioned invariance and unknown-input observation for two-dimensional FornasiniMarchesini models. In Proceedings of European Control Conference, Kos, 2007.

[23] R.P. Roesser. A discrete state-space model for linear image processing. IEEE Transactions on Automatic Control, vol. AC-20, no. 1, pp. 1-10, February 1975.

[24] H.L. Trentelman, A.A. Stoorvogel, and M. Hautus. Control theory for linear systems. Communications and Control Engineering. Springer, Great Britain, 2001.

[25] J. Willems, Almost invariant subspaces: an approach to high gain feedback design - Part II: Almost conditionally invariant subspaces, IEEE Transactions on Automatic Control, vol. AC-26, no. 1, pp. 235-252, 1981.

[26] W.M. Wonham and A.S. Morse. Decoupling and pole assignment 
in linear multivariable systems: a geometric approach. SIAM Journal of Control, vol. 8, no. 1, pp. 1-18, 1970.

[27] T. Zhou. Stability and Stability Margin for a Two-Dimensional System. IEEE Transactions on Signal Processing, vol. 54, no. 9, pp. 3483-3488, September 2006. 